\title{
Broadband quarter-wave plates at near-infrared using high-contrast gratings
}

Mehmet Mutlu

Ahmet E. Akosman

Gokhan Kurt

Mutlu Gokkavas

Ekmel Ozbay 


\title{
Broadband quarter-wave plates at near-infrared using high-contrast gratings
}

\author{
Mehmet Mutlu, ${ }^{a, b}$ Ahmet E. Akosman, ${ }^{a, b}$ Gokhan Kurt, ${ }^{b}$ \\ Mutlu Gokkavas, ${ }^{b}$ and Ekmel Ozbay ${ }^{a, b, c}$ \\ ${ }^{a}$ Department of Electrical and Electronics Engineering, Bilkent University, Ankara, Turkey; \\ ${ }^{b}$ Nanotechnology Research Center, Bilkent University, Ankara, Turkey; \\ ${ }^{c}$ Department of Physics, Bilkent University, Ankara, Turkey
}

\begin{abstract}
In this paper, we report the theoretical and experimental possibility of achieving a quarter-wave plate regime by using high-contrast gratings, which are binary, vertical, periodic, near-wavelength, and two-dimensional high refractive index gratings. Here, we investigate the characteristics of two distinct designs, the first one being composed of silicon-dioxide and silicon, and the second one being composed of silicon and sapphire. The suggested quarter-wave plate regime is achieved by the simultaneous optimization of the transverse electric and transverse magnetic transmission coefficients, $T_{\mathrm{TE}}$ and $T_{\mathrm{TM}}$, respectively, and the phase difference between these transmission coefficients, such that $\left|T_{\mathrm{TM}}\right| \simeq\left|T_{\mathrm{TE}}\right|$ and $\angle T_{\mathrm{TM}}-\angle T_{\mathrm{TE}} \simeq \pi / 2$. As a result, a unity circular polarization conversion efficiency is achieved at $\lambda_{0}=1.55 \mu \mathrm{m}$ for both designs. For the first design, we show the obtaining of unity conversion efficiency by using a theoretical approach, which is inspired by the periodic waveguide interpretation, and rigorous coupled-wave analysis (RCWA). For the second design, we demonstrate the unity conversion efficiency by using the results of finite-difference time-domain (FDTD) simulations. Furthermore, the FDTD simulations, where material dispersion is taken into account, suggest that an operation percent bandwidth of $51 \%$ can be achieved for the first design, where the experimental results for the second design yield a bandwidth of $33 \%$. In this context, we define the operation regime as the wavelength band for which the circular conversion efficiency is larger than 0.9 .
\end{abstract}

Keywords: Polarization, periodic slab waveguide, high-contrast grating, quarter-wave plate

\section{INTRODUCTION}

High-contrast gratings (HCGs), which are composed of high refractive index gratings with subwavelength periodicities enclosed by low refractive index materials, ${ }^{1}$ have been notably studied since the numerical ${ }^{2}$ and experimental ${ }^{3}$ revelation of their diffraction-free and broadband high-reflectivity regimes. The most notable and intriguing properties of HCGs include large fabrication tolerance, geometrical simplicity and design flexibility. ${ }^{4}$ In addition, their well-established theoretical descriptions also contribute to the enabling of benefiting from HCGs for the purpose of designing various optical devices, i.e., polarization-independent broadband reflectors, ${ }^{5}$ polarizing beam splitters, ${ }^{6}$ saturable and cavity-enhanced absorbers,,${ }^{7,8}$ monolithic high-reflectivity cavity mirrors, ${ }^{9}$ planar lenses and reflectors with high focusing abilities, ${ }^{10,11}$ high quality-factor Fabry-Perot resonators ${ }^{12}$ vertical-cavity surface emitting and nanoelectromechanical lasers, ${ }^{13,14}$ slow light waveguides, ${ }^{15}$ and one-, twoand three- dimensional hollow-core low-loss optical waveguides. ${ }^{16-18}$

In this study, we benefit from the unique properties of HCGs for the purpose of achieving the desired transmission characteristics of a broadband and highly efficient quarter-wave plate, which operates in the nearinfrared regime. It has recently been shown that transmission characteristics of binary and vertical HCGs can be obtained by the implementation of the periodic dielectric slab waveguide interpretation. ${ }^{19}$ In one of our recent studies, we propose a two-dimensional HCG structure with optimized geometrical parameters ${ }^{20}$ by

Further author information: (Send correspondence to M. Mutlu)

M. Mutlu: E-mail: mutlu@ee.bilkent.edu.tr, Telephone: +90 3122901019

E. Ozbay: E-mail: ozbay@bilkent.edu.tr, Telephone: +90 3122901966

High Contrast Metastructures II, edited by Connie J. Chang-Hasnain, Fumio Koyama,

Alan Eli Willner, Weimin Zhou, Proc. of SPIE Vol. 8633, 863300 · ( 2013 SPIE

CCC code: $0277-786 X / 13 / \$ 18 \cdot$ doi: $10.1117 / 12.2009347$

Proc. of SPIE Vol. $8633863300-1$ 


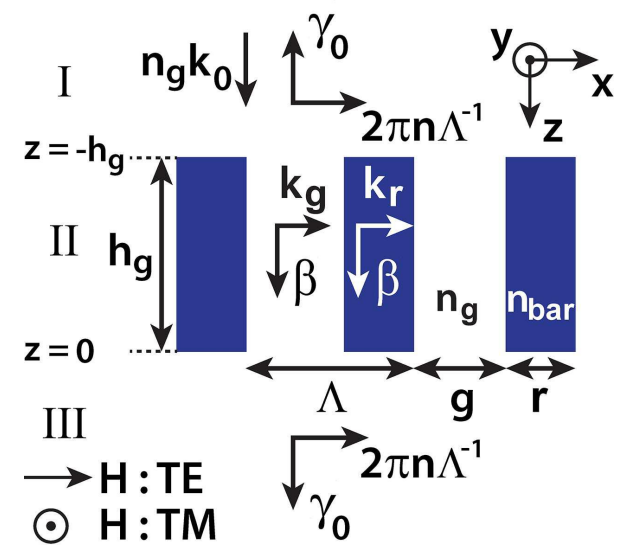

Figure 1: Geometrical description of the proposed HCG based quarterwave plate. The incident wave is impinged on the structure from region I, where the transmitted converted wave propagates in region III.

benefiting from this theoretical consideration, rigorous coupled-wave analysis ${ }^{21}$ and finite-difference time domain simulations (FDTD Solutions, Lumerical Inc.). After presenting the detailed theoretical investigation of the HCG based quarter-wave plate, we have recently demonstrated the experimental realization of another HCG based quarter-wave plate by proposing a structure with a more feasible choice of materials. ${ }^{22}$

For the theoretical study (first design), the transmission results obtained from the RCWA and FDTD based simulations reveal that an operation bandwidth of $54 \%$ and $51 \%$ can be achieved, respectively, under the assumption that the structure is in the operation regime if the conversion efficiency is equal to or larger than 0.9. Likewise, an operation bandwidth of $42 \%$ and $33 \%$ is achieved from the FDTD simulations and measurements, respectively, in the experimental study (second design). Further, it should be noted that such designs are promising for the implementation of compact and electrically thin circular polarizers by combining the proposed quarter-wave structures with HCG based, ${ }^{6}$ wire grid, ${ }^{23}$ thin-film grating, ${ }^{24}$ or plasmonic ${ }^{25}$ linear polarizers.

\section{ANALYSIS OF THE FIRST DESIGN}

Firstly, we propose a HCG based structure, for which the aim is to maximize the operation bandwidth and total transmission with a reasonable design concerning the practical realization stage. The proposed design is depicted in Fig. 1. For the achievement of a realistic design, the material constituting region III is selected as silicon-dioxide $\left(\mathrm{SiO}_{2}\right)$. Furthermore, as a result of aiming the maximization of the operation band and the transmission through the structure, the material constituting region I and the grooves in region II is selected as also $\mathrm{SiO}_{2}$. Finally, we select the material for the ridges as silicon $(\mathrm{Si})$ due to its high refractive index and low absorption coefficient at $\lambda_{0}=1.55 \mu \mathrm{m}$.

Concerning the theoretical consideration and numerical simulations, regions I and III are assumed to be extending to infinity in $-z$ and $+z$ directions, respectively. In order to approximate this assumption in the experimental realization stage, one can use a substrate (in region III) and deposit a dielectric layer (in region I) that is much thicker than the coherence length of the incident light.

In the subsequent subsections, we will first consider the proposed HCG theoretically, and then present circular conversion coefficients and the circular conversion efficiency spectrum.

\subsection{Theoretical Analysis}

For the purpose of analyzing the proposed HCG structure theoretically, we adopt the analysis that is inspired by the periodic waveguide interpretation and performed by Karagodsky et al. ${ }^{19}$ Accordingly, we obtain the 
following condition for the unity transmission of a TM wave that is normally incident onto the structure:

$$
\left|\sum_{m}\left(a_{m}^{\mathrm{TM}}-a_{m}^{\rho, \mathrm{TM}}\right) \Lambda^{-1} \int_{0}^{\Lambda} h_{y, m}^{i n, \mathrm{TM}}(x) d x\right|=1,
$$

where $a_{m}^{\mathrm{TM}}$ and $a_{m}^{\rho, \mathrm{TM}}$ represent the coefficients of the magnetic field components of the $m^{\text {th }}$ TM waveguide mode propagating in the $+z$ and $-z$ directions inside region II, respectively. $\Lambda$ is the period of the HCG structure, and $h_{y, m}^{i n, T M}$ is the lateral magnetic field distribution of the corresponding TM mode. By invoking the duality principle, the unity transmission condition for TE waves can be simply written as follows:

$$
\left|\sum_{m}\left(a_{m}^{\mathrm{TE}}-a_{m}^{\rho, \mathrm{TE}}\right) \Lambda^{-1} \int_{0}^{\Lambda} h_{x, m}^{i n, \mathrm{TE}}(x) d x\right|=1 .
$$

For the achievement of the quarter-wave plate operation, i.e., transmission of a circularly polarized wave when the structure is illuminated by a linearly polarized wave with a polarization plane angle of $\pi / 4$ with respect to the $x$-axis on the $x y$-plane, it is necessary to also satisfy the following condition:

$$
\angle T_{\mathrm{TM}}-\angle T_{\mathrm{TE}} \simeq \pi / 2 .
$$

In Eq. 3, TM and TE can be interchanged and the choice is arbitrary. In this case, our choice leads to the transmission of a right hand circularly polarized wave when the polarization plane angle of the incident wave is $\pi / 4$. The operation condition given in Eq. 3 corresponds to the following equation in the theoretical consideration:

$$
\angle\left(\sum_{m}\left(a_{m}^{\mathrm{TM}}-a_{m}^{\rho, \mathrm{TM}}\right) \Lambda^{-1} \int_{0}^{\Lambda} h_{y, m}^{i n, \mathrm{TM}}(x) d x\right)-\angle\left(\sum_{m}\left(a_{m}^{\mathrm{TE}}-a_{m}^{\rho, \mathrm{TE}}\right) \Lambda^{-1} \int_{0}^{\Lambda} h_{x, m}^{i n, \mathrm{TE}}(x) d x\right)= \pm \pi / 2 .
$$

Afterwards, the longitudinal wavenumber of the waveguide modes inside region II, $\beta_{m}$, can be written as a function of the incident wavelength and the lateral wavenumbers as follows:

$$
\beta_{m}^{2}=\left(2 \pi n_{g} / \lambda_{0}\right)^{2}-k_{g, m}^{2}=\left(2 \pi n_{b a r} / \lambda_{0}\right)^{2}-k_{r, m}^{2},
$$

where $\lambda_{0}$ is the free-space wavelength, and $k_{g, m}$ and $k_{r, m}$ are the lateral wavenumbers inside $\mathrm{SiO}_{2}$ and $\mathrm{Si}$, respectively. Subsequently, for TM modes, the dispersion equation, the relation between the lateral wavenumbers, is given as follows:

$$
n_{b a r}^{-2} k_{r, m} \tan \left(k_{r, m} r / 2\right)=-n_{g}^{-2} k_{g, m} \tan \left(k_{g, m} g / 2\right) .
$$

By invoking the duality principle, the dispersion relation for TE modes can be written as:

$$
k_{r, m} \tan \left(k_{r, m} r / 2\right)=-k_{g, m} \tan \left(k_{g, m} g / 2\right) .
$$

The qualitative interpretation of Eqs. 6 and 7 is that the TM and TE waveguide modes can have different lateral wavenumbers, which, according to Eq. 5, results in different longitudinal wavenumbers. As a consequence, one can expect the TE and TM modes to accumulate different phases upon transmission through the HCG.

For the purpose of calculating the transmission coefficients of TE and TM waves, we invoke a two mode approximation at this point, i.e., introducing the restriction that $m$ can be 0 and 1 . Under this approximation, the longitudinal and lateral wavenumbers for TM and TE modes can be calculated using Eqs. 5 and 6, and Eqs. 5 and 7 , respectively. In the calculations, $n_{b a r}=3.48$ and $n_{g}=1.47$, which correspond to the refractive indices of $\mathrm{Si}$ and $\mathrm{SiO}_{2}$, respectively, at the frequency of interest, $\lambda_{0}=1.55 \mu \mathrm{m}$. Afterwards, with the help of a custom parametric optimization code, we obtain the geometrical parameters, for which Eqs. 1, 2, and 4 are satisfied simultaneously, by using the following definitions for $h_{y, m}^{i n, \mathrm{TM}}$ and $h_{x, m}^{i n, \mathrm{TE}}:{ }^{19}$

$$
h_{y, m}^{i n, \mathrm{TM}}=h_{x, m}^{i n, \mathrm{TE}}=\left\{\begin{array}{ll}
\cos \left(k_{r, m} r / 2\right) \cos \left[k_{g, m}(x-g / 2)\right], & \text { inside the grooves } \\
\cos \left(k_{g, m} g / 2\right) \cos \left\{k_{r, m}[x-(g+\Lambda) / 2]\right\}, & \text { inside the ridges }
\end{array} .\right.
$$


Accordingly, the optimal geometric parameters for quarter-wave plate operation at $\lambda_{0}=1.55 \mu \mathrm{m}$ are obtained as $r=160 \mathrm{~nm}, g=220 \mathrm{~nm}$, and $h_{g}=550 \mathrm{~nm}$. The lateral wavenumbers of TM modes for these geometric parameters are calculated as $k_{g, 0}^{2}=-4.2 / \Lambda^{2}, k_{r, 0}^{2}=19.43 / \Lambda^{2}$ and $k_{g, 1}^{2}=31.3 / \Lambda^{2}, k_{r, 1}^{2}=54.9 / \Lambda^{2}$. Similarly, for TE modes, we obtain $k_{g, 0}^{2}=-13.1 / \Lambda^{2}, k_{r, 0}^{2}=10.48 / \Lambda^{2}$ and $k_{g, 1}^{2}=30.2 / \Lambda^{2}, k_{r, 1}^{2}=53.8 / \Lambda^{2}$. Recalling that, in such a waveguide, the cutoff condition is given by $k_{r}^{2}=\left(n_{b a r} / n_{g}\right)^{2} k_{g}^{2}$ and thus, it is seen that only the first modes in the TM and TE cases are propagating modes with $\beta_{m}^{2}>0$. The second modes in both cases correspond to exponentially decaying modes with $\beta_{m}^{2}<0$. In addition, the longitudinal wavenumbers of the diffraction orders is given by $\gamma_{n}^{2}=\left(2 \pi n_{g}\right)^{2}\left(\lambda_{0}^{-2}-n^{2} \Lambda^{-2}\right)$, where $n$ denotes the diffraction order. Since $\lambda_{0} / \Lambda=4.1$ in the proposed design, it follows that $\gamma_{0}^{2}>0$, where $\gamma_{n}^{2}<0$ for $\forall n, n \neq 0$. In other words, only $0^{\text {th }}$ reflection and transmission orders are propagating, which results in the fact that, if a planewave propagating in the $+z$ direction is impinged on the structure, the reflected and transmitted waves are planewaves propagating in the $-z$ and $+z$ directions, respectively.

Finally, we calculate the complex transmission coefficients, $T_{\mathrm{TM}}$ and $T_{\mathrm{TE}}$, theoretically. We obtain $\left|T_{\mathrm{TM}}\right|=$ 0.988 and $\left|T_{\mathrm{TE}}\right|=0.936$, where $\angle T_{\mathrm{TM}}-\angle T_{\mathrm{TE}}=92^{\circ}$. The resulting transmission coefficients for the aforementioned geometrical parameters suggest that the desired quarter-wave operation regime is achieved theoretically. For detailed information regarding the calculation of the transmission coefficients, we suggest the readers to refer to Ref. 19.

\subsection{Numerical Validation}

For the purpose of validating our theoretical predictions, we employ numerical simulations based on RCWA and FDTD method. Using these methods, $T_{\mathrm{TM}}$ and $T_{\mathrm{TE}}$ are calculated. In RCWA, we neglect the material dispersion, whereas we take the material dispersion into account properly in the FDTD method. Subsequenty, the circular conversion coefficients, $C_{+}$(for right-hand circularly polarized waves) and $C_{-}$(for left hand circularly polarized waves), which indicate the amplitudes and phases of the circularly polarized waves at the output interface for an incident plane wave with a polarization plane angle of $\pi / 4$, can be directly calculated by a simple transformation of the basis vectors as $C_{ \pm}=0.5\left(T_{\mathrm{TM}} \mp i T_{\mathrm{TE}}\right){ }^{26}$

For being able to measure the degree of conversion from the linear polarization to the circular one, we define the conversion efficiency parameter, $C_{\text {eff }}$, as follows:

$$
C_{\text {eff }}=\frac{\left|C_{+}\right|^{2}-\left|C_{-}\right|^{2}}{\left|C_{+}\right|^{2}+\left|C_{-}\right|^{2}}
$$

It is noteworthy that the ellipticity parameter, $\eta$, can be directly calculated from the $C_{\text {eff }}$ spectrum as follows:

$$
\eta=\arctan \left(\frac{\sqrt{\left(1+C_{\text {eff }}\right) /\left(1-C_{\text {eff }}\right)}-1}{\sqrt{\left(1+C_{\text {eff }}\right) /\left(1-C_{\text {eff }}\right)}+1}\right) .
$$

Using the relation given in Eq. 10, it can be shown that $C_{\text {eff }}=0.9$ corresponds to $\eta=32^{\circ}$. Due to the one-to-one correspondence between $C_{\text {eff }}$ and $\eta$, we only provide $C_{\text {eff }}$ in this paper.

The circular conversion coefficients obtained from RCWA and the conversion efficiency spectrum obtained from RCWA and FDTD simulations are shown in Fig. 2. The presented results obtained from RCWA suggest that a conversion efficiency that is larger than 0.9 is achieved between $1.36 \mu \mathrm{m}$ and $2.36 \mu \mathrm{m}$. The FDTD results suggest a narrower wavelength band, $1.4 \mu \mathrm{m}-2.36 \mu \mathrm{m}$, as a consequence of the dependence of the refractive index of $\mathrm{Si}$ on the wavelength. For the calculation of the percent bandwidth of operation, we use the following classical definition: ${ }^{27}$

$$
\mathrm{BW} \%=200 \% \frac{\lambda_{H} / \lambda_{L}-1}{\lambda_{H} / \lambda_{L}+1},
$$

where $\lambda_{H}$ and $\lambda_{L}$ are the higher and lower corner wavelengths of the operation band, respectively. Using the definition given in Eq. 11, we obtain BW\% $=54 \%$ and BW\% $=51 \%$ from the RCWA and FDTD results, respectively. 

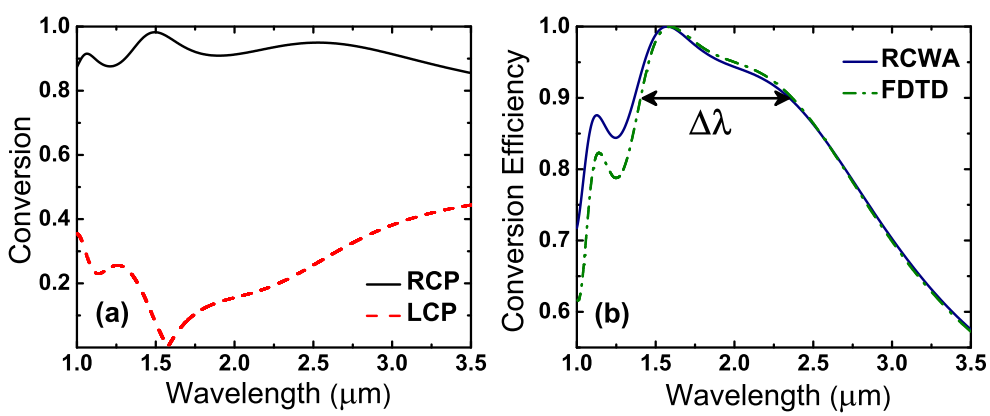

Figure 2: (a) Circular conversion coefficients obtained using the RCWA and (b) the conversion efficiencies obtained from the RCWA and FDTD. The wavelength range for the FDTD result satisfying the 0.9 efficiency threshold is denoted by $\Delta \lambda$.

\section{ANALYSIS OF THE SECOND DESIGN}

After demonstrating the possibility of a HCG based quarter-wave plate in Section 2, we modify the design depicted in Fig. 1 such that the choice of materials is more feasible for fabrication. In this design, the material filling region III is sapphire, whereas region I and the grooves in region II are filled with free-space.

For the present design, a theoretical interpretation is not provided since it is largely similar to the one shown in Section 2.1. However, one major difference arises from the choice of different materials for region I and III. In the present case, the longitudinal wavenumber of the zeroth reflection order is given by $\gamma_{0, \mathrm{I}}=2 \pi \lambda_{0}^{-1}$, where, for the zeroth transmission order, we have $\gamma_{0, \mathrm{III}}=2 \pi n_{s} \lambda_{0}^{-1}$. This characteristic conduces to discrepant lateral magnetic field distributions in regions I and III. Thus, one should pay attention to the utilization of correct distributions while matching the tangential electric and tangential magnetic fields at $z=0$ and $z=-h_{g}$.

\subsection{Numerical Results}

The geometrical parameters of the HCG structure for optimal operation, i.e., unity conversion efficiency at $\lambda_{0}=1.55 \mu \mathrm{m}$, are obtained by utilizing the theoretical model and RCWA simulations as $r=220 \mathrm{~nm}, g=350 \mathrm{~nm}$, and $h_{g}=320 \mathrm{~nm}$. Subsequently, FDTD simulations are run in order to characterize the transmission of the
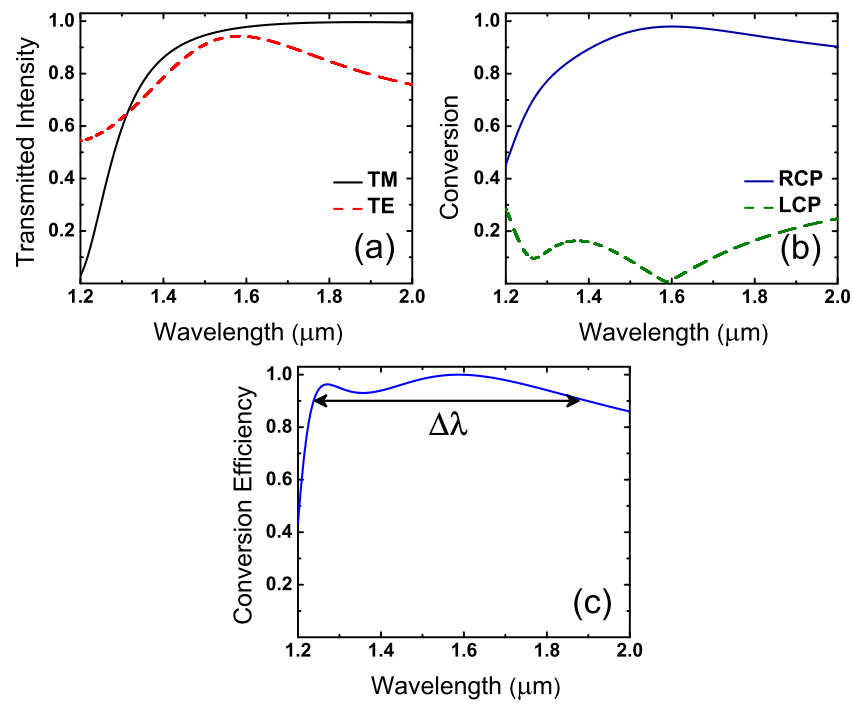

Figure 3: (a) Circular conversion coefficients obtained using the RCWA and (b) the conversion efficiencies obtained from the RCWA and FDTD. The wavelength range for the FDTD result satisfying the 0.9 efficiency threshold is denoted by $\Delta \lambda$. 


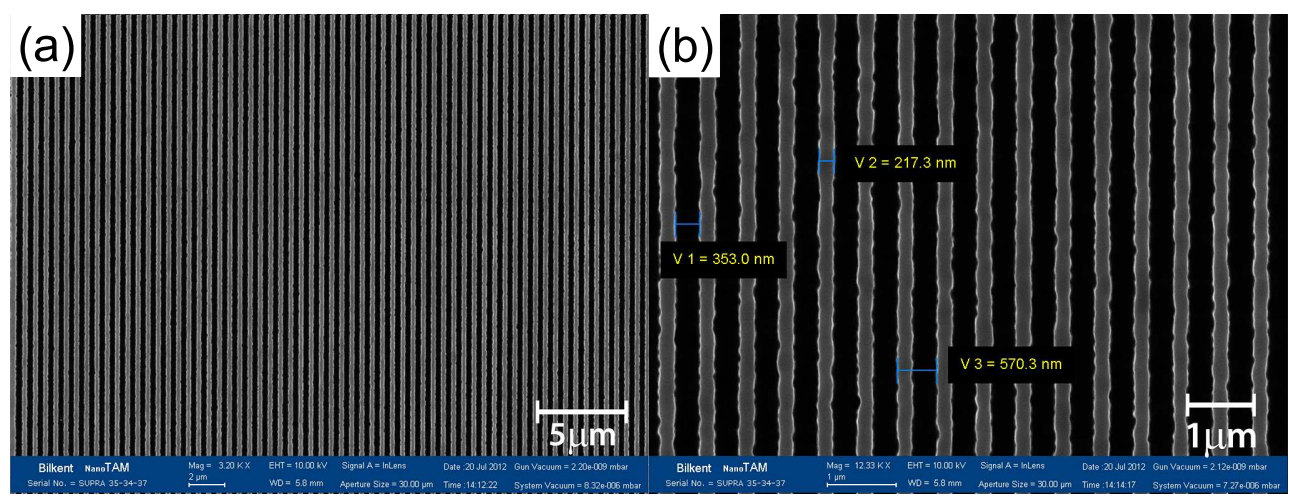

Figure 4: (a) Zoomed out and (b) zoomed in top view SEM micrographs of the fabricated HCG structure. In (b), the legends V1, V2, and V3 denote the geometrical parameters g, r, and, respectively.

proposed structure. The numerically obtained normalized transmitted intensities $\left(\left|T_{\mathrm{TM}}\right|^{2}\right.$ and $\left.\left|T_{\mathrm{TE}}\right|^{2}\right)$ are shown in Fig. 3(a).

In the close neighborhood of $\lambda_{0}=1.55 \mu \mathrm{m}$, the conditions given in Eqs. 1, 2, and 4 are satisfied simultaneously, which result in the transmission of a right hand circularly polarized wave assuming that a planewave with a polarization plane angle of $\pi / 4$ is impinged on the structure. The conversion coefficients are calculated using the same formulation given in Sec. 2.2 and the results are shown in Fig. 3(b). Finally, the conversion efficiency spectrum is calculated using Eq. 9 and shown in Fig. 3. The numerical results suggest that the condition $C_{\text {eff }}>0.9$ is satisfied in the wavelength interval $1.24 \mu \mathrm{m}-1.40 \mu \mathrm{m}$. By using Eq. 11, it is calculated that this wavelength interval corresponds to a percent bandwidth of $42 \%$.

\subsection{Fabrication}

The proposed HCG based quarter-wave plate is fabricated using a silicon-on-sapphire wafer (Valley Design Corporation). The thickness of the sapphire (R-plane) substrate is specified as $0.52 \pm 0.05 \mathrm{~mm}$. The $<100>$ Si layer is grown epitaxially on the substrate and its thickness is $600 \pm 60 \mathrm{~nm}$. The fabrication of the structure starts with the reduction of the thickness of the Si layer to $320 \mathrm{~nm}$ by using sulfur hexafluoride chemistry based reactive ion etching (RIE). Afterwards, an electron beam sensitive photoresist, poly(methyl methacrylate), is deposited on the wafer by means of spinning. After photoresist deposition, the desired regions are exposed by the e-beam lithography technique. The e-beam step is followed by the development of the photoresist. Finally, the unexposed Si regions are etched with the aid of the RIE system. The scanning electron microscope micrographs of the fabricated HCG structure are shown in Fig. 4.

\subsection{Experimental Setup}

The detailed outlines of the two experimental setups are depicted in Fig. 5. The first setup, see Fig. 5(a), is utilized for measuring the intensity transmission coefficients, $\left|T_{\mathrm{TM}}\right|^{2}$ and $\left|T_{\mathrm{TE}}\right|^{2}$. In this measurement, the sample is illuminated by a broadband near-infrared light source (Spectral Products, ASBN-W100-F-L) and the polarization of the incident wave is set to either TM or TE polarization by the usage of an adjustable linear polarizer (Thorlabs, DGL10). The spot size of the incident light on the sample is set to $50 \mu \mathrm{m}$ with the aid of standard commercial objectives. The transmitted light is collimated by the objectives at the output interface and coupled into the fiber. Finally, the spectrum of the transmitted wave is analyzed by the spectrometer (Ocean Optics, NIR256-2.5).

The second setup, see Fig. 5(b), is utilized for the measurement of the circular conversion coefficients, $C_{+}$ and $C_{-}$. Considering that the linear polarizer at the input interface makes an angle of $45^{\circ}$ with the $x$-axis on the $x y$-plane and the quarter-wave plate (Thorlabs, WPQ05M-1550) is oriented such that its fast axis is along the $x$ direction, the HCG is illuminated by a right hand circularly polarized wave. Using Jones calculus, the 

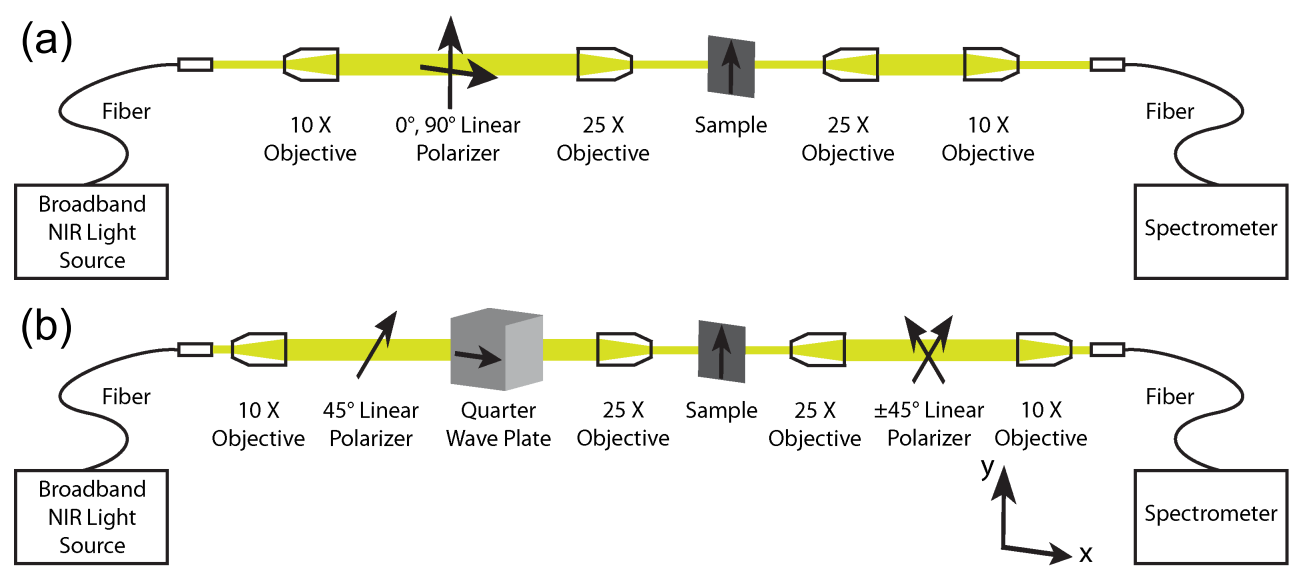

Figure 5: Visual illustrations of the experimental setups that are utilized for the measurement of (a) linear transmission and (b) circular conversion coefficients.

transmission characteristics of the HCG can be described as follows:

$$
\left(\begin{array}{l}
E_{x}^{t} \\
E_{y}^{t}
\end{array}\right)=\left(\begin{array}{cc}
T_{\mathrm{TM}} & 0 \\
0 & T_{\mathrm{TE}}
\end{array}\right)\left(\begin{array}{l}
E_{x}^{i} \\
E_{y}^{i}
\end{array}\right),
$$

where $E^{i}$ and $E^{t}$ denote the incident and transmitted fields, respectively. Therefore, by aligning the linear polarizer transmission axis at $-45^{\circ}$ and $45^{\circ}$, we measure $\left|C_{+}\right|^{2}$ and $\left|C_{-}\right|^{2}$, respectively. For instance, it is seen that, if the structure is illuminated by a right hand circularly polarized wave with unity amplitude at $\lambda_{0}=1.55 \mu \mathrm{m}$, the transmitted wave will be represented as $(1 / \sqrt{2} ;-1 / \sqrt{2})$ and passed by a polarizer with its transmission axis aligned at $-45^{\circ}$. Similarly, at $\lambda_{0}=1.55 \mu \mathrm{m}$, a linear polarizer oriented at $45^{\circ}$ passes the transmitted wave, $(1 / \sqrt{2} ; 1 / \sqrt{2})$, when the structure is illuminated by a left hand circularly polarized wave with unity amplitude.
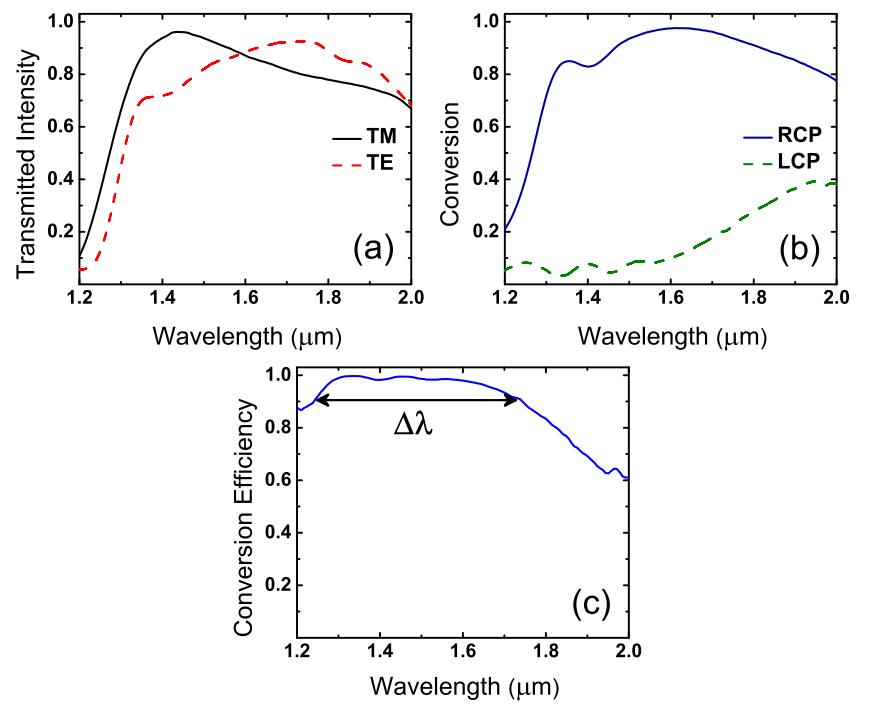

Figure 6: Experimentally obtained (a) TM and TE transmitted intensities, (b) circular conversion coefficients, and (c) conversion efficiency spectrum. The wavelength interval of operation is denoted by $\Delta \lambda$. The experimental conversion efficiency spectrum yields a percent bandwidth of $33 \%$. 


\subsection{Experimental Results}

The linear intensity transmission coefficients, $\left|T_{\mathrm{TM}}\right|^{2}$ and $\left|T_{\mathrm{TE}}\right|^{2}$, measured using the setup shown in Fig. 5(a) are shown in Fig. 6(a). The circular conversion coefficients measured using the setup shown in Fig. 5(b) are shown in Fig. 6(b). Finally, the conversion efficiency spectrum, $C_{\text {eff }}$, is calculated from the circular conversion coefficients by using Eq. 9 and plotted in Fig. 6(c). The experimental $C_{\text {eff }}$ spectrum suggests that the lower and higher corner wavelengths are $\lambda_{L}=1.25 \mu \mathrm{m}$ and $\lambda_{H}=1.75 \mu \mathrm{m}$, respectively, which result in a percent bandwidth of $33 \%$. Although the experimental bandwidth is smaller compared to the numerical one, $42 \%$, the experimental results are in good agreement with the numerical ones.

\section{CONCLUSIONS}

In conclusion, we have shown the theoretical realization and the experimental characterization of broad-band quarter-wave plates that are composed of two-dimensional HCGs. While the material chosen for the ridges remain same both in theoretical and experimental studies, the material used for the substrate is changed to Sapphire from $\mathrm{SiO}_{2}$ for the purpose of achieving a more fabricable design. For the first design, it is shown that a conversion efficiency that is higher than 0.9 can be achieved in theoretical and numerical percent bandwidth of $54 \%$ and $51 \%$, respectively. Similarly, a percent bandwidth of $42 \%$ and $33 \%$ is achieved numerically and experimentally for the second design. The possible application areas of the proposed quarter-wave plates can include liquid crystal displays, laser applications and remote sensors.

\section{ACKNOWLEDGMENTS}

The authors would like to thank Adil Burak Turhan for the e-beam lithography process and Yasemin Kanli for the initial FTIR measurements. This work is supported by the projects DPT-HAMIT, ESF-EPIGRAT and NATO-SET-181, and TUBITAK under the Project Nos. 107A004, 109A015, and 109E301. One of the authors (E. Ozbay) also acknowledges partial support from the Turkish Academy of Sciences.

\section{REFERENCES}

[1] Karagodsky, V. and Chang-Hasnain, C. J., "Physics of near-wavelength high contrast gratings," Opt. Express 20(10), 10888-10895 (2012).

[2] Mateus, C., Huang, M., Deng, Y., Neureuther, A., and Chang-Hasnain, C., "Ultrabroadband mirror using low-index cladded subwavelength grating," IEEE Photonic Tech. Lett. 16(2), 518 -520 (2004).

[3] Mateus, C., Huang, M., Chen, L., Chang-Hasnain, C., and Suzuki, Y., "Broad-band mirror (1.12-1.62 $\mu \mathrm{m})$ using a subwavelength grating," IEEE Photonic Tech. Lett. 16(7), 1676 -1678 (2004).

[4] Zhou, Y., Huang, M., and Chang-Hasnain, C., "Large fabrication tolerance for VCSELs using high-contrast grating," IEEE Photonic Tech. Lett. 20(6), $434-436$ (2008).

[5] Wu, H., Mo, W., Hou, J., Gao, D., Hao, R., Jiang, H., Guo, R., Wu, W., and Zhou, Z., "A high performance polarization independent reflector based on a multilayered configuration grating structure," J. Opt. 12(4), 045703 (2010).

[6] Wu, H., Mo, W., Hou, J., Gao, D., Hao, R., Guo, R., Wu, W., and Zhou, Z., "Polarizing beam splitter based on a subwavelength asymmetric profile grating," J. Opt. 12(1), 015703 (2010).

[7] Yang, W., Sedgwick, F., Zhang, Z., and Chang-Hasnain, C. J., "High contrast grating based saturable absorber for mode-locked lasers," Conference on Lasers and Electro-Optics , CThI5, Optical Society of America (2010).

[8] Zohar, M., Auslender, M., Faraone, L., and Hava, S., "Novel resonant cavity-enhanced absorber structures for high-efficiency midinfrared photodetector application," J. Nanophoton. 5(1), 051824 (2011).

[9] Brückner, F., Friedrich, D., Clausnitzer, T., Britzger, M., Burmeister, O., Danzmann, K., Kley, E.-B., Tünnermann, A., and Schnabel, R., "Realization of a monolithic high-reflectivity cavity mirror from a single silicon crystal," Phys. Rev. Lett. 104(16), 163903 (2010).

[10] Lu, F., Sedgwick, F. G., Karagodsky, V., Chase, C., and Chang-Hasnain, C. J., "Planar high-numericalaperture low-loss focusing reflectors and lenses using subwavelength high contrast gratings," Opt. Express 18(12), 12606-12614 (2010). 
[11] Fattal, D., Li, J., Peng, Z., Fiorentino, M., and Beausoleil, R. G., "Flat dielectric grating reflectors with focusing abilities," Nat. Photon. 4(7), 466-470 (2010).

[12] Karagodsky, V., Chase, C., and Chang-Hasnain, C. J., "Matrix Fabry-Perot resonance mechanism in highcontrast gratings," Opt. Lett. 36(9), 1704-1706 (2011).

[13] Huang, M. C. Y., Zhou, Y., and Chang-Hasnain, C. J., "A surface-emitting laser incorporating a highindex-contrast subwavelength grating," Nat. Photon. 1(2), 119-122 (2007).

[14] Huang, M. C. Y., Zhou, Y., and Chang-Hasnain, C. J., "A nanoelectromechanical tunable laser," Nat. Photon. 2(3), 180-184 (2008).

[15] Karagodsky, V., Pesala, B., Sedgwick, F. G., and Chang-Hasnain, C. J., "Dispersion properties of highcontrast grating hollow-core waveguides," Opt. Lett. 35(24), 4099-4101 (2010).

[16] Zhou, Y., Karagodsky, V., Pesala, B., Sedgwick, F. G., and Chang-Hasnain, C. J., "A novel ultra-low loss hollow-core waveguide using subwavelength high-contrast gratings," Opt. Express 17(3), 1508-1517 (2009).

[17] Yue, Y., Zhang, L., Wang, X., Huang, H., Yang, W., Ferrara, J., Karagodsky, V., Chase, C., Tur, M., ChangHasnain, C., and Willner, A., "Three-dimensional chirped high-contrast grating hollow-core waveguide," IEEE Photonic Tech. Lett. 4(5), $1372-1380$ (2012).

[18] Huang, H., Yue, Y., Zhang, L., Chase, C., Parekh, D., Sedgwick, F., Wu, M., Chang-Hasnain, C., Tur, M., and Willner, A., "Analog signal transmission in a high-contrast-gratings-based hollow-core-waveguide," $J$. Lightwave Technol. 30(23), 3640 -3646 (2012).

[19] Karagodsky, V., Sedgwick, F. G., and Chang-Hasnain, C. J., "Theoretical analysis of subwavelength high contrast grating reflectors," Opt. Express 18(16), 16973-16988 (2010).

[20] Mutlu, M., Akosman, A. E., and Ozbay, E., "Broadband circular polarizer based on high-contrast gratings," Opt. Lett. 37(11), 2094-2096 (2012).

[21] Moharam, M. G., Grann, E. B., Pommet, D. A., and Gaylord, T. K., "Formulation for stable and efficient implementation of the rigorous coupled-wave analysis of binary gratings," J. Opt. Soc. Am. A 12(5), 10681076 (1995).

[22] Mutlu, M., Akosman, A. E., Kurt, G., Gokkavas, M., and Ozbay, E., "Experimental realization of a highcontrast grating based broadband quarter-wave plate," Opt. Express 20(25), 27966-27973 (2012).

[23] Ahn, S.-W., Lee, K.-D., Kim, J.-S., Kim, S. H., Park, J.-D., Lee, S.-H., and Yoon, P.-W., "Fabrication of a $50 \mathrm{~nm}$ half-pitch wire grid polarizer using nanoimprint lithography," Nanotechnology 16(9), 1874-1877 (2005).

[24] So, D. W. C. and Seshadri, S. R., "Thin-film grating polarizer," Opt. Lett. 19(7), 469-471 (1994).

[25] Schider, G., Krenn, J. R., Gotschy, W., Lamprecht, B., Ditlbacher, H., Leitner, A., and Aussenegg, F. R., "Optical properties of Ag and Au nanowire gratings," J. Appl. Phys. 90(8), 3825-3830 (2001).

[26] Mutlu, M., Akosman, A. E., Serebryannikov, A. E., and Ozbay, E., "Asymmetric chiral metamaterial circular polarizer based on four U-shaped split ring resonators," Opt. Lett. 36(9), 1653-1655 (2011).

[27] Balanis, C. A., [Antenna Theory: Analysis and Design], Wiley (2005). 